\title{
Spin-dependent transport in a quasiballistic quantum wire
}

\author{
C.-T. Liang \\ Cavendish Laboratory, Madingley Road, Cambridge CB3 OHE, United Kingdom \\ and Department of Physics, National Taiwan University, Taipei 106, Taiwan \\ M. Pepper \\ Cavendish Laboratory, Madingley Road, Cambridge CB3 OHE, United Kingdom \\ M. Y. Simmons \\ Cavendish Laboratory, Madingley Road, Cambridge CB3 OHE, United Kingdom \\ and Semiconductor Nanofabrication Facility, School of Physics, University of New South Wales, Sydney 2052, Australia \\ C. G. Smith and D. A. Ritchie \\ Cavendish Laboratory, Madingley Road, Cambridge CB3 OHE, United Kingdom \\ (Received 1 September 1999; revised manuscript received 25 October 1999)
}

\begin{abstract}
We describe the transport properties of a 5- $\mu \mathrm{m}$-long one-dimensional (1D) quantum wire. Reduction of conductance plateaus due to the introduction of weak disorder scattering is observed. In an in-plane magnetic field, we observe a spin splitting of the reduced conductance steps. Our experimental results provide evidence that the deviation from conductance quantization is very small for electrons with spin parallel and is about $1 / 3$ for electrons with spin antiparallel. Moreover, in a high in-plane magnetic field, a spin-polarized 1D channel shows a plateaulike structure close to $0.3 e^{2} / h$ which strengthens with increasing temperature. It is suggested that these results arise from a combination of disorder and electron-electron interactions in a 1D electron gas.
\end{abstract}

Using electron beam lithography, one is able to pattern the surface of a GaAs/AlGaAs heterostructure with submicron Schottky gates. By negatively biasing the surface gates, one can electrostatically squeeze ${ }^{1}$ the underlying twodimensional electron gas (2DEG) into various shapes. The most noteworthy success of this technique is the experimental realization of a one-dimensional (1D) channel — by using a pair of "split gates,", it is possible to define a 1D channel within a $2 \mathrm{DEG} .^{2}$ If the elastic scattering length is longer than the $1 \mathrm{D}$ channel length, transport through the channel is ballistic and one observes conductance plateaus quantized in units of $2 e^{2} / h .^{3,4}$ Although $1 \mathrm{D}$ electron transport has been studied for more than a decade, most experimental results can be explained within a single-particle picture without considering electron-electron interactions and spin effects in a $1 \mathrm{D}$ system. It is only more recently that a " 0.7 structure,', evidence for possible spin polarization caused by electronelectron interactions, ${ }^{5}$ has been extensively studied. Part of the reason may be that in a clean system the conductance is independent of the electron-electron interactions and it is determined by entrance and exit reservoirs. ${ }^{6}$ A noninteracting 1D system should show either ballistic quantization or have a conductance decreasing to zero with increasing length and decreasing temperature due to localization. On the other hand it has been suggested that the interaction is observable in the presence of backscattering, ${ }^{7}$ so called a dirty Luttinger liquid, but excess disorder may remove all semblance of 1D transport.

It is well known that as the length of a quantum wire is increased, the effect of disorder within the wire is enhanced ${ }^{8}$ and backscattering in the channel is increased, resulting in a crossover from ballistic towards diffusive transport. In this paper, we present experimental results on the transport properties of a 5- $\mu \mathrm{m}$ quantum wire. In such a regime the weak disorder within the system reduces the conductance steps below quantized units in $2 e^{2} / h$. In an in-plane magnetic field, we observed a spin splitting of the conductance plateaus, as expected. Recently Kimura, Kuroki, and Aoki ${ }^{9}$ have proposed that in a dirty Luttinger liquid, a reduction of spinantiparallel conductance occurs. That is, due to the electronelectron interactions, the conductance for spin-antiparallel electrons is smaller than that for spin-parallel electrons. We shall show that our results are consistent with their model. When there are more than one 1D subbands occupied in the channel, our data further suggest that the electron transmission probability through a long quasiballistic channel shows oscillating behavior with electron spin species. Moreover, below the first spin-polarized conductance step, a plateaulike structure close to $0.3 e^{2} / h$ strengthens with increasing temperature.

The split-gate (SG) device ( $5 \mu \mathrm{m}$ long and $0.8 \mu \mathrm{m}$ wide) was lithographically defined, $300 \mathrm{~nm}$ above the 2DEG. The 2DEG has a carrier density of $3 \times 10^{11} \mathrm{~cm}^{-2}$ with a mobility of $7.5 \times 10^{6} \mathrm{~cm}^{2} / \mathrm{V} \mathrm{s}$ after brief illumination with a red light emitting diode. Experiments were performed in a pumped ${ }^{3} \mathrm{He}$ cryostat and the two-terminal conductance $G=d I / d V$ was measured using an ac excitation voltage of $10 \mu \mathrm{V}$ at a frequency of $77 \mathrm{~Hz}$ with standard phase-sensitive techniques. The in-plane magnetic field $B_{\|}$is applied parallel to the source-drain current. To check for an out-of-plane magnetic field component, we measure the Hall voltage. From this we know that the sample was aligned better than $0.1^{\circ}$ using an in situ rotating insert. In all cases, a zero-split-gate-voltage series resistance due to the bulk 2DEG is subtracted from the 


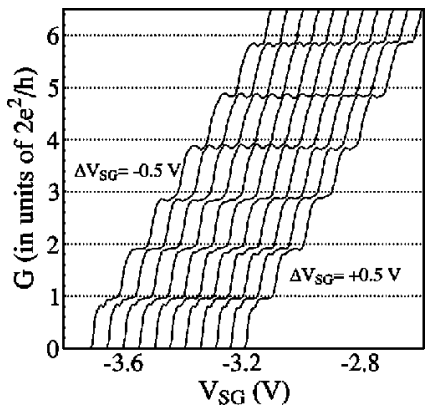

FIG. 1. $G\left(V_{S G}\right)$ measurements when the channel is laterally shifted by differentially biasing the two halves of the split gates. From left to right: $\Delta V_{S G}=-0.5 \mathrm{~V}$ to $+0.5 \mathrm{~V}$ in $0.1 \mathrm{~V}$ steps. The measurement temperature was $0.3 \mathrm{~K}$.

raw data. In the literature, it has been shown that there is an additional series resistance between a $1 \mathrm{D}$ channel and bulk 2DEG. ${ }^{10}$ Three different samples at four cooldowns show similar behavior and measurements taken from one of these samples are presented in this paper.

Impurities in the spacer layer can give rise to potential fluctuations ${ }^{11}$ near a $1 \mathrm{D}$ wire. This effect could cause nonuniformity of the quantum wire confinement potential, leading to a microconstriction, a narrowest region in the channel. To check whether conduction through a 1D channel is dominated by a microconstriction, one can laterally shift the conduction channel. ${ }^{12}$ When the channel is moved away from a microconstriction, the conductance-split-gate-voltage pinchoff characteristics would show large variations because of the sudden disappearance of the extra confining potential due to impurities which causes the microconstriction in the channel. We now show that it is not the case in our long quantum wire. Figure 1 shows $G\left(V_{S G}\right)$ when we differentially bias the two halves of the split gates. In this case, the 1D channel is laterally shifted by $\pm 57 \mathrm{~nm} .{ }^{13}$ It is evident that the pinch-off voltages show a linear dependence of the voltage difference between the two halves of the split gate. Also, resonant features and conductance plateaus show gradual evolution as the channel is moved laterally. This demonstrates that transport through the channel is not dominated by a narrow region in the channel. As shown in Fig. 1, resonant features superimposed upon conductance steps are clearly observed in all

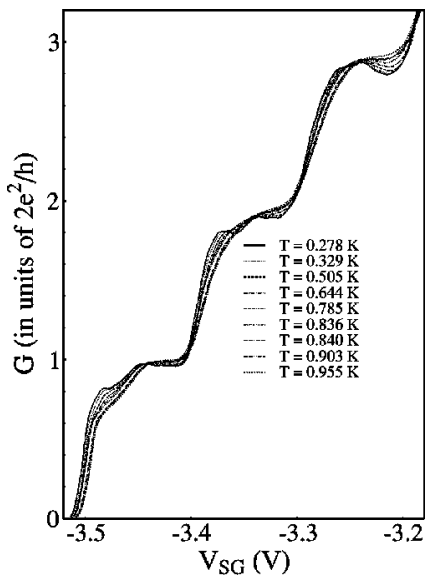

FIG. 2. $G\left(V_{S G}\right)$ at various temperatures $T$ as illustrated in the figure.

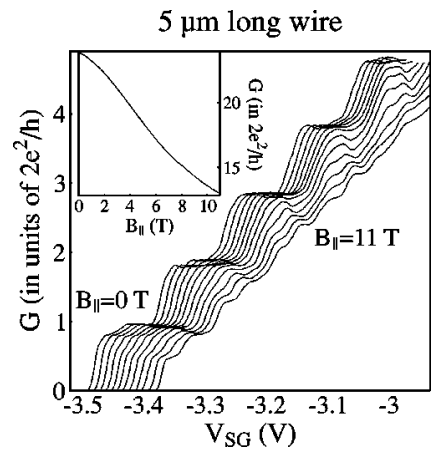

FIG. 3. $G\left(V_{S G}\right)$ at various applied magnetic fields parallel to the 2DEG $B_{\|}$. From left to right: $B_{\|}=0$ to $11 \mathrm{~T}$ in $1 \mathrm{~T}$ steps. Curves are successively offset by $0.01 \mathrm{~V}$ for clarity. The zero-split-gate-voltage conductance at various $B_{\|}$, as shown in the inset, has been subtracted from the raw data. The inset shows the zero-split-gatevoltage conductance as a function of $B_{\|}$. The measurement temperature was $0.3 \mathrm{~K}$.

traces. These resonances are believed to be due to potential fluctuations near the $1 \mathrm{D}$ channel. $^{8}$ The scattering potential within the channel does indeed vary when the 1D channel is moved laterally as the strength of resonant features changes, as illustrated in Fig. 1. Nevertheless, note that conductance plateau deviations from their quantized values are always observed in all 11 traces. However it is noticeable that the plateaus are reasonably intact, showing that the scattering is weak and does not vary significantly with the Fermi energy so producing a semblance of plateaus. In this paper, we concentrate on the case where there is no potential difference between the two halves of the SG.

Figure 2 shows conductance-split-gate-voltage characteristics $G\left(V_{S G}\right)$ at various temperatures $T$. With increasing $T$, the feature close to $0.8 \times 2 e^{2} / h$ and resonant features gradually disappear. The first three conductance plateau values increase and approach multiples of $2 e^{2} / h$ at the highest temperatures. In a shorter wire $(3 \mu \mathrm{m})$, clean conductance plateaus close to multiples of $2 e^{2} / h$ are observed. With increasing temperature, the conductance plateaus become less well defined due to thermal smearing. Nevertheless, the midpoints of the conductance steps remain close to multiples of $2 e^{2} / h$ at high temperatures. This effect is not related to the reports of decreased plateau values. For example, recently the role of electron injection into $\mathrm{V}$-groove quantum wires has been studied. ${ }^{14}$ It has been shown that the observed reduction of ballistic conductance steps is due to poor coupling between the 1D states of the wire and the 2D states of the reservoirs. This mechanism may account for the reduced conductance plateaus observed in cleaved edge overgrown quantum wires studied by Yacoby and co-workers, ${ }^{15}$ which is entirely different from the spin-dependent effects previously reported. ${ }^{5}$

We now turn our attention to the reduced conductance plateaus as a function of magnetic field applied parallel to the 2DEG $B_{\|}$. It is well established that a large $B_{\|}$lifts the electron spin degeneracy as demonstrated by Wharam et al., ${ }^{3}$ causing consecutive spin-parallel (parallel to $B_{\|}$) and spinantiparallel (antiparallel to $B_{\|}$) conductance plateaus in multiples of $e^{2} / h{ }^{16}$ Figure 3 shows $G\left(V_{S G}\right)$ at various $B_{\|}$. With increasing $B_{\|}$, the splitting of the conductance steps can be seen and the spin-split conductance step values are somewhat lower than multiples of $e^{2} / h$. It is worth mentioning 


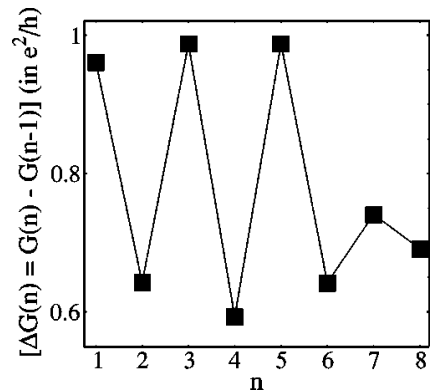

FIG. 4. The difference in conductance between the $(n-1)$ th and the $n$th $1 \mathrm{D}$ conductance steps $\Delta G(n)=G(n)-G(n-1)$, where $n$ is the spin-split subband index.

that the feature close to $0.8 \times 2 e^{2} / h$, believed to be due to resonant transmission through an impurity potential, ${ }^{17,18}$ gradually disappears with increasing $B_{\|}$. This result, together with the data shown in Fig. 1 when the feature close to $0.8 \times 2 e^{2} / h$ gradually turns into a resonant peak as the channel is laterally shifted, shows that at zero magnetic field one needs to be careful in ascribing any feature close to $0.7 \times 2 e^{2} / h$ observed in a $1 \mathrm{D}$ channel to the " 0.7 plateau" extensively studied by Thomas and co-workers. ${ }^{5}$ The zerosplit-gate-voltage conductance shows a monotonic decrease with increasing $B_{\|}$, as illustrated in the inset to Fig. 3. This effect is due to the diamagnetic shift of the 2DEG. ${ }^{19}$

As clearly shown in Fig. 3, at $B_{\|}=11 \mathrm{~T}$ the conductance does not show steps in multiples of $e^{2} / h$. We now use a different view which reveals a striking behavior. Figure 4 now shows the difference in conductance between the midpoints of consecutive steps value $\Delta G(n)=G(n)-G(n-1)$ where $n$ is the number of spin-split 1D subbands occupied. For $n=1, \Delta G(1)$ is simply the conductance step value. For $n \leqslant 6$, an oscillating behavior is evident $-\Delta G(n)$ approaches a quantized value of $e^{2} / h$ when $n$ is an odd integer, and shows substantial deviations (up to $1 / 3$ ) from a quantized value of $e^{2} / h$ when $n$ is an even integer. For $n \geqslant 6$, the conductance steps are less pronounced and the striking oscillating behavior gradually disappears. Assuming that $\Delta G(n)$ reflects the transmission probability for the $n$th spin-split 1D subband, then these experimental results suggest that the spin-parallel electrons have almost a full transmission probability $(100 \%)$ through the 1D channel whereas the spinantiparallel electrons have a much lower transmission probability $(\approx 65 \%)$. The semblance of the observed 1D conductance steps, together with the observed weak resonant features in our weakly disordered $1 \mathrm{D}$ wire, suggests that our device is in the dirty Luttinger liquid regime. If this is the case, then our experimental results are consistent with the model proposed by Kimura and co-workers. ${ }^{9}$ Note that in their model they only consider a two-band (spin-parallel and spin-antiparallel electrons) Tomonaga-Luttinger liquid. The fact that the reduction of spin-antiparallel conductance persists up to $n=6$ suggests that our results can be extended to a "six-band" (three pairs of spin-parallel and spinantiparallel electrons) limit.

Finally we present the temperature dependence measurements which reveal even more striking behavior. Figure 5 shows $G\left(V_{S G}\right)$ for $B_{\|}=11 \mathrm{~T}$ at different temperatures. As expected, the spin-split conductance steps become less pro-

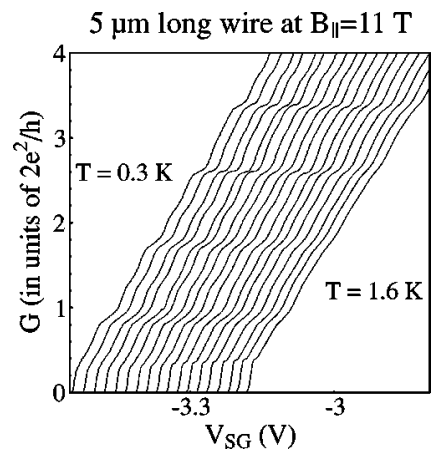

FIG. 5. $G\left(V_{S G}\right)$ for $B_{\|}$at various temperatures $T$. From left to right: $T=0.3,0.363,0.391,0.429,0.483,0.542,0.611,0.680$, $0.752,0.832,0.920,1.01,1.07,1.16,1.28,1.46$, and $1.60 \mathrm{~K}$. Curves are successively offset by $0.01 \mathrm{~V}$ for clarity. The data were taken at the second cooldown.

nounced at higher temperatures due to thermal broadening. However, a plateaulike structure close to $0.3 e^{2} / h$ becomes more pronounced with increasing temperatures. Also the structure approaches $0.4 e^{2} / h$ at the highest temperature. The reason for this unexpected behavior is not fully understood at present but we speculate that strong electron-electron interactions might play a role in this.

The oscillating, spin-dependent transmission probability which we observed is in striking contrast to the behavior of a short, "point contact" ballistic channel where the quantization is always in units of $e^{2} / h$ regardless of spin orientation. In the latter case, theory shows that due to mixing of the reservoir and channel states there can be no electron-electron interaction-enhanced deviation from the quantized values. However, Kimura and co-workers ${ }^{9}$ have considered the Tomonaga-Luttinger liquid when backscattering occurs; they find that this mixes with the interaction to produce a conductance dependent on the Fermi energy and hence a spin state although it is still surprising the spin-parallel electrons display a quantized value. Recently it has been suggested that in the Tomonaga-Luttinger regime charge density wave formation can give rise to a fractional charge behavior. ${ }^{20}$ The results here are consistent with the spin-antiparallel electrons showing a quantized conductance but with the value of the fundamental charge reduced. We note that we cannot attribute the spin-dependent behavior to any spin-dependent scattering at the entrance and the exit to the channel as it is absent from short devices of the same heterostructure material and the effect is reproducible despite a change in sample and channel location.

In summary, we have performed low-temperature measurements on a quasiballistic quantum wire. Our results suggest that the electron transmission probability through a long quasiballistic channel shows oscillating behavior with spin species. Moreover, a spin-polarized 1D channel shows a pronounced plateaulike structure close to $0.3 e^{2} / h$ with increasing strength at higher temperatures. Such striking behavior is only observed in long quantum wires $(\approx 5 \mu \mathrm{m})$ but not in a clean 1D channel $(\leqslant 3 \mu \mathrm{m})$, suggesting that a combination of backscattering and interactions within the quasiballistic 1D system plays an important role. 
This work was funded by the UK EPSRC and, in part, by the U.S. Army Research Office. We thank C.J.B. Ford for helpful discussions, K.J. Thomas for drawing our attention to Ref. 9, H.D. Clark, J.E.F. Frost, and M. Kataoka for advice and help on device fabrication at an early stage of this work, and S. Shapira for experimental assistance. C.T.L. is grateful for support from the Department of Physics, National Taiwan University.
${ }^{1}$ T.J. Thornton et al., Phys. Rev. Lett. 56, 1198 (1986).

${ }^{2}$ K.-F. Berggren, T.J. Thornton, D.J. Newson, and M. Pepper, Phys. Rev. Lett. 57, 1769 (1986).

${ }^{3}$ D.A. Wharam et al., J. Phys. C 21, L209 (1988).

${ }^{4}$ B.J. van Wees et al., Phys. Rev. Lett. 60, 848 (1988).

${ }^{5}$ K.J. Thomas et al., Phys. Rev. Lett. 77, 135 (1996); Phys. Rev. B 58, 4846 (1998).

${ }^{6}$ D.L. Maslov and M. Stone, Phys. Rev. B 52, R5539 (1995).

${ }^{7}$ D.L. Maslov, Phys. Rev. B 52, R14 368 (1995).

${ }^{8}$ J.A. Nixon, J.H. Davies, and H.U. Baranger, Phys. Rev. B 43, 12638 (1991).

${ }^{9}$ T. Kimura, K. Kuroki, and H. Aoki, Phys. Rev. B 53, 9572 (1996).

${ }^{10} \mathrm{G}$. Timp et al., in Proceedings of the International Symposium on Nanostructure Physics and Fabrication, College Station, Texas, edited by M.A. Reed and W.P. Kirk (Academic, New York, 1989), pp. 331-345.

${ }^{11}$ J. A. Nixon and J. H. Davies, Phys. Rev. B 41, 7929 (1990).

${ }^{12}$ R.J. Stroh and M. Pepper, J. Phys.: Condens. Matter 1, 8481 (1989).

${ }^{13}$ J.G. Williamson et al., Phys. Rev. B 42, 7675 (1990).

${ }^{14}$ C.-T. Liang et al., Appl. Phys. Lett. 75, 2975 (1999).

${ }^{15}$ A. Yacoby et al., Phys. Rev. Lett. 77, 4612 (1996).

${ }^{16}$ N.K. Patel et al., Phys. Rev. B 44, R10 973 (1991).

${ }^{17}$ J.T. Nicholls et al., Phys. Rev. B 48, 8866 (1993).

${ }^{18}$ C.-T. Liang et al., Phys. Rev. B 55, 6723 (1997).

${ }^{19}$ J. Weis et al., Surf. Sci. 305, 664 (1994).

${ }^{20}$ V.V. Ponomarenko and N. Nagaosa, Phys. Rev. Lett. 83, 1822 (1999). 